\title{
Adherencia al tratamiento en la insuficiencia cardiaca y las tecnologías de la información y la comunicación
}

\author{
Carolina Hernández-Pinzón ${ }^{\mathrm{a}, *}$ y Magda L. Flórez-Flórez ${ }^{\mathrm{b}}$ \\ a Unidad de Insuficiencia Cardiaca, Clínica Universitaria Colombia, Bogotá, Colombia \\ ${ }^{\mathrm{b}}$ Facultad de Enfermería, Universidad Nacional de Colombia, Bogotá, Colombia
}

Recibido el 30 de enero de 2016; aceptado el 10 de julio de 2016

Disponible en Internet el 30 de septiembre de 2016

\author{
PALABRAS CLAVE \\ Insuficiencia \\ cardiaca; \\ Adhesión; \\ Tratamiento
}

\begin{abstract}
Resumen
Objetivo: Determinar la efectividad del uso de las tecnologías de la información y la comunicación, en la adherencia al tratamiento de los pacientes con insuficiencia cardiaca, que asistieron a una Unidad de Insuficiencia Cardiaca de Bogotá.

Métodos: Se realizó un ensayo clínico con preprueba, posprueba y grupo de control. Se conformaron aleatoriamente tres grupos de 37 participantes cada uno, a dos de los cuales se les aplicó una intervención durante dos meses basada en el uso de las tecnologías de la información y la comunicación; el grupo 1 recibió seguimiento telefónico más envío de mensajes de texto, el grupo 2 seguimiento telefónico más envío de correos electrónicos y el grupo 3 correspondiente al grupo de control recibió la intervención de enfermería usual en una Unidad de Insuficiencia Cardiaca.

Resultados: Los tres grupos definidos para el estudio modificaron significativamente sus niveles medios de adherencia; en los grupos 1 y 2 dicho incremento es atribuible a la intervención con el uso de las tecnologías de la información y la comunicación, de igual manera la adherencia en los participantes del grupo control que recibieron la atención usual en una Unidad de Insuficiencia Cardiaca, también muestra un incremento significativo.

Conclusiones: El uso de las tecnologías de la información y la comunicación: el seguimiento telefónico asociado a los mensajes de texto y los correos electrónicos incrementó la adherencia al tratamiento farmacológico y no farmacológico en pacientes con insuficiencia cardiaca a través de la motivación y el seguimiento de los pacientes.

(C) 2016 Sociedad Colombiana de Cardiología y Cirugía Cardiovascular. Publicado por Elsevier España, S.L.U. Este es un artículo Open Access bajo la licencia CC BY-NC-ND (http:// creativecommons.org/licenses/by-nc-nd/4.0/).
\end{abstract}

\footnotetext{
* Autor para correspondencia.

Correo electrónico: chernandezp@unal.edu.co (C. Hernández-Pinzón).
} 


\section{KEYWORDS}

Heart failure;

Adherence;

Treatment

\section{Treatment adherence in heart failure and information and communication technologies}

\begin{abstract}
Motivation: To determine the efficacy of the use of information and communication technologies in the treatment adherence of patients with heart failure that were assisted at the Heart Failure Unit of Bogotá.

Methods: A clinical trial was conducted with pre-, post-test and control group. Three randomised groups of 37 participants each were conformed, two of which received an intervention during two months based in the use of information and communication technology; group 1 received telephone follow-up plus text messages, group 2 telephone follow-up plus emails and group 3, which was the control group, received the usual nurse follow-up at a Heart Failure Unit.

Results: The three defined study groups significantly modified their average adherence levels; groups 1 and 2 the increase is attributable to the intervention with the use of information and communication technologies, likewise, adherence of the control group participants who received the usual care at a Heart Failure Unit also showed a significant increase.

Conclusion: The use of information and communication technologies, such as the telephone follow-up associated to text messages and emails, rose the adherence to the pharmacological and non-pharmacological treatment in patients with heart failure by motivating and monitoring patients.

(c) 2016 Sociedad Colombiana de Cardiología y Cirugía Cardiovascular. Published by Elsevier España, S.L.U. This is an open access article under the CC BY-NC-ND license (http:// creativecommons.org/licenses/by-nc-nd/4.0/).
\end{abstract}

\section{Introducción}

Las enfermedades cardiovasculares son en la actualidad una de las principales causas de la morbilidad y la mortalidad a nivel mundial ${ }^{1}$, la insuficiencia cardiaca hace parte de esta categoría, su prevalencia e incidencia se incrementan con la edad ${ }^{2}$ y se ha convertido en el problema de salud pública que afecta en gran medida a los individuos, a sus familias y a la sociedad.

La insuficiencia cardiaca es una enfermedad crónica no trasmisible de gran interés para los profesionales de enfermería teniendo en cuenta su impacto en la salud de los individuos, genera: discapacidad, presencia de síntomas como disnea, fatiga y edemas ${ }^{3}$, que afectan la realización de sus actividades de la vida diaria representado en deterioro de su calidad de vida ${ }^{4}$. Adicionalmente, repercute en el sistema de salud en costos y altas tasas de morbi-mortalidad, convirtiéndose en un problema de salud pública a nivel nacional y mundial $^{5}$.

En la actualidad existen tratamientos que han demostrado ser efectivos en el control de la insuficiencia cardiaca, no obstante, se ha demostrado en la literatura que el déficit en el autocuidado expresado en la baja adherencia a los tratamientos no permite alcanzar las metas propuestas en el manejo de dicha patología 6 .

Las intervenciones que se han realizado incluyen diferentes estrategias, un ejemplo de ellas es la creación de unidades multidisciplinarias de la insuficiencia cardiaca ${ }^{7}$, en donde los profesionales de la enfermería participan activamente a través de los programas de educación estructurados, el seguimiento telefónico y el uso de las tecnologías de la información y la comunicación ${ }^{8}$.

En este marco es indispensable y de gran impacto en la adherencia al tratamiento, la educación estructurada, la monitorización, el seguimiento y la motivación de estos pacientes, donde las tecnologías de la información y la comunicación se convierten en herramientas novedosas para el logro de estas metas.

Dentro de estas tecnologías innovadoras y de bajo costo para el sistema de salud que permiten ser empleadas en este grupo de pacientes, se encuentran el envío de mensajes de texto y los correos electrónicos, que han demostrado tener un impacto, especialmente, en la reducción de ingresos hospitalarios, adherencia al tratamiento y reconocimiento temprano de descompensaciones a través del seguimiento y la motivación de los pacientes ${ }^{9-12}$, no obstante, su uso debe ser combinado con una tecnología que permita establecer un canal de comunicación no presencial con el paciente como lo es el seguimiento telefónico de los pacientes, que fomente la relación terapéutica entre el paciente y el profesional de enfermería, evitando que exista una brecha entre los pacientes y el personal de salud en razón a que los mensajes de texto y los correos electrónicos son herramientas digitales y dependen del acceso de las personas a dichas tecnologías.

Este estudio aporta conocimiento en relación al uso y efectividad de las tecnologías de la información y la comunicación, se convierte en producción científica que permite relacionar el cuidado de la enfermería, la práctica y la investigación en el contexto de la insuficiencia cardiaca en Colombia.

\section{Métodos}

Diseño experimental tipo ensayo clínico en el cual se manipuló la variable independiente (intervención de la enfermería basada en el uso de las tecnologías de la información y la comunicación) para determinar su efecto en 
la variable dependiente (adherencia al tratamiento de los pacientes con insuficiencia cardiaca).

Se conformaron aleatoriamente tres grupos de 37 participantes, el grupo uno recibió la intervención de seguimiento telefónico más el envío de mensajes de texto, el grupo dos recibió la intervención de seguimiento telefónico más el envío de correos electrónicos y el grupo control recibió la atención usual en la Unidad de Insuficiencia Cardiaca.

La población del estudio estuvo conformada por pacientes con diagnóstico de insuficiencia cardiaca, según guías y protocolos institucionales, que asistían a la consulta ambulatoria programada.

Se utilizó la siguiente expresión algebraica para el cálculo del tamaño de la muestra de cada grupo, que hace referencia a la pauta propuesta por Schwertman en la determinación del tamaño requerido para sustentar el juzgamiento de la hipótesis nula de igualdad de promedios, bajo la admisión de homoscedasticidad y la condición del tamaño balanceado de las muestras.

Esta expresión corresponde a:

$n=2\left\{\sqrt{(k-1)-(k-2)}+z_{1-\beta}\right\}^{2}\left(\frac{\sigma}{\Delta}\right)^{2}$

Donde, $n$ : El tamaño de la muestra de cada grupo.

$\alpha$ : La probabilidad de error tipo I.

$\beta$ : La probabilidad de error tipo ॥. $k$ : El número de grupos.

$(k-1)$ : El percentil $100(1-\alpha)$ de una distribución chicuadrado con $(k-1)$ grados de libertad.

$z_{1-}$ : El percentil $100(1-\beta)$ de una distribución normal estándar.

$\sigma$ : La desviación estándar de la variable de interés.

$\Delta$ : Diferencia máxima entre promedios para la consideración $\operatorname{de} \beta$.

Se realizó el cálculo del tamaño de la muestra a partir de la medición de la adherencia en la preprueba asumiendo los siguientes valores: probabilidad de error tipo । $5 \%(0,05)$ y probabilidad de error tipo ॥ $2 \%(0,02)$ con una diferencia mínima entre promedios de 10 puntos de la escala y una desviación estándar de 9,1 estimada a partir de la información del puntaje obtenido en la medición de adherencia basal de los primeros 100 participantes en el estudio.

Se definió entonces el tamaño de la muestra en donde cada grupo de intervención y el grupo de control deberían estar conformados por 30 participantes, y considerando el porcentaje de pérdida del $20 \%$, se adicionaron 7 participantes a cada grupo, por tanto, el tamaño de la muestra fue de 111 participantes. Los criterios de inclusión de los participantes para ingresar al estudio fueron: ser mayores de 18 años de edad, existencia de un cuidador que realizara el acompañamiento durante las intervenciones en los casos que fuera necesario por limitaciones físicas del paciente de acceso al teléfono, mensajes de texto y/o correos electrónicos, pacientes que recibieran la intervención usual de enfermería en la Unidad de Insuficiencia Cardiaca a través de la consulta de enfermería, paciente o su cuidador con acceso a un teléfono celular y acceso al internet. Dentro de los criterios de exclusión se establecieron: deterioro cognitivo grave documentado en la historia clínica según evaluación realizada por la psicóloga del equipo multidisciplinario y pacientes que no aceptaron el grupo al cual fueron asignados (intervención-control).
Para la asignación aleatoria se realizó un muestreo probabilístico de los pacientes que asistían a las consultas programadas con el grupo multidisciplinario en la Unidad de Insuficiencia Cardiaca. Los pacientes que cumplieron los criterios de inclusión fueron aleatorizados para la asignación tanto al grupo de intervención como al grupo control.

Para la asignación aleatoria se elaboró una lista de 111 números aleatorios en Excel con la función aleatorio entre 1 y 3 , es decir, el paciente se asignaba al grupo 1 de intervención si el resultado de la función mencionada era 1 , al grupo 2 de intervención si el resultado la de función mencionada era 2 y al grupo 3 control si el resultado de la función mencionada era 3 . Esta función genera números aleatorios provenientes de una distribución uniforme discreta, es decir, se generan con igual probabilidad. La aleatorización de los participantes fue realizada por un auxiliar de la investigación, para garantizar la asignación en los diferentes grupos de la investigación.

La evaluación de la adherencia se realizó con el instrumento de evaluación de la adherencia al tratamiento farmacológico y no farmacológico en los pacientes con falla cardiaca $^{13}$, el cual está conformado por 10 dimensiones e incluye en total 30 ítems con cuatro posibles respuestas, con una escala tipo Likert (nada, muy poco, frecuentemente, mucho). La sumatoria total, determina la adherencia en rangos: no adherente 30-55 puntos, poca adherencia 56-80 puntos, frecuentemente adherido 81-105 puntos, adherido 106-120 puntos, fue aplicado a todos los participantes antes de iniciar la intervención y al finalizar la misma.

La asignación aleatoria y la medición de la adherencia fueron realizadas por dos auxiliares de la investigación, quienes no conocían a los participantes y que fueron capacitados por la investigadora principal, según las recomendaciones de la autora del instrumento.

Se diseñó un formulario digital para la obtención de los datos del estudio en el cual se incorporaron las variables de medición y se ingresaron los datos correspondientes a las variables sociodemográficas, clínicas y los datos obtenidos de la aplicación del instrumento, a través de un dispositivo portátil (tablet) con conexión al internet, que permitía ingresar las respuestas en tiempo real y de esta manera alimentar directamente la matriz de datos del estudio.

\section{Procedimiento}

Una vez obtenido el aval ético por parte del Comité de Ética de la Facultad de Enfermería de la Universidad Nacional de Colombia y la Unidad de Investigaciones de la Fundación Universitaria Sanitas se inició la recolección de la muestra.

A continuación, se describe la Fase 1 de la investigación que corresponde al ingreso de los participantes al estudio.

\section{Fase 1. Ingreso al estudio de los pacientes}

Se invitó a cada participante al estudio, indicándole que sería asignado aleatoriamente a uno de los grupos de intervención o control.

De acuerdo al grupo al cual fueran asignados los participantes, se procedió a la lectura y firma del consentimiento informado, el auxiliar de la investigación indicó a aquellos que hacían parte del grupo 1 (seguimiento telefónico más 
mensajes de texto) y grupo 2 (seguimiento telefónico más correos electrónicos) y a los participantes del grupo 3 (control), que recibirían la atención usual de enfermería.

Posteriormente, el auxiliar de la investigación realizó la aplicación del formulario digital en donde se ingresaron los datos correspondientes a las variables sociodemográficas, clínicas y los datos obtenidos de la aplicación del instrumento de evaluación de adherencia de los pacientes antes de la intervención.

Se informó a todos los participantes que debían asistir a la intervención usual de enfermería y/o a recibir atención prioritaria en la Unidad de Insuficiencia Cardiaca en caso de requerirlo.

\section{Fase 2. Intervención}

Para la realización de la intervención se elaboraron, con base en la literatura revisada, los contenidos educativos, de seguimiento y motivacionales que fueron entregados a los participantes en el seguimiento telefónico, mensajes de texto y correos electrónicos.

Seguimiento telefónico: durante 2 meses se realizaron en total 5 seguimientos telefónicos con intervalo de 15 días entre cada uno, en cada llamada se evaluó el estado de salud actual, si se habían presentado síntomas de descompensación, si había consultado al servicio de urgencias y si había requerido hospitalizaciones, se preguntó a cada participante si había recibido el correo electrónico o el mensaje de texto, según el caso, enviados durante esa semana y se comunicaron los contenidos educativos para cada llamada.

Mensajes de texto y correo electrónico: se envió un mensaje de texto o correo semanalmente durante 2 meses, mensajes de texto (grupo 1) o correos electrónicos (grupo 2 ), con contenido educativo, motivacional y de seguimiento.

En los grupos de intervención la comunicación fue bidireccional, para mantener contacto con los pacientes en el caso en que ellos lo necesitaran, a través de la línea telefónica destinada para tal fin.

Los participantes del grupo de intervención en los cuales se identificó algún riesgo de descompensación, fueron atendidos de inmediato en la Unidad de Insuficiencia Cardiaca. Todos los participantes del estudio seguían recibiendo la atención usual de educación y atención por el grupo multidisciplinario.

Los pacientes pertenecientes al grupo 3 (grupo control) recibieron la intervención de enfermería usual en la unidad de insuficiencia cardiaca, esta intervención se realizó por medio de consultas de enfermería programadas, según protocolo institucional.

Una vez finalizada la intervención, el auxiliar de investigación aplicó el formulario digital en donde se ingresaron los datos correspondientes a la aplicación del instrumento de evaluación de adherencia de los pacientes, se realizó a través de llamadas telefónicas de acuerdo a la disponibilidad de los pacientes y teniendo en cuenta que los pacientes manifestaron dificultades para acceder a la unidad de insuficiencia cardiaca.

La investigadora principal conocía a los participantes y realizó la intervención. En la firma del consentimiento informado los pacientes eran asignados a los grupos y se les indicaba a cual grupo pertenecían. Por tanto, debido a la naturaleza y ética del estudio no se estableció ninguna clase de cegamiento, sumado a que las intervenciones en enfermería son totalmente visibles contrario a los ensayos clínicos puros.

\section{Control de sesgos del estudio}

Con la finalidad de evitar la aparición de errores sistemáticos durante la selección o el seguimiento de la población en el estudio, se conformaron tres grupos de comparación con el mismo número de participantes en cada grupo, la asignación de los participantes a los grupos se realizó de forma aleatoria para que existiera equivalencia de grupos al inicio y durante la intervención, lo que permitió asegurar la conformación de grupos con características similares, excepto en la intervención, y de igual manera las variables intervinientes quedaron igualmente distribuidas en los grupos, evitando que afectaran de forma sistemática el estudio. El estudio contó con un grupo de control para determinar la relación causal entre las variables independiente y dependiente y contener la influencia de variables externas.

El sesgo del entrevistador se puede presentar teniendo en cuenta que la investigadora conocía a los participantes, por tal motivo la asignación aleatoria y la medición de adherencia en la pre y pos prueba, fue realizada por dos auxiliares de la investigación, quienes no conocían a los participantes, antes de iniciar la intervención y al finalizar la misma.

Se buscó controlar la instrumentación, entrenando dos auxiliares de la investigación en la aplicación del instrumento.

\section{Resultados}

La muestra final del estudio estuvo conformada por 97 pacientes con la insuficiencia cardiaca que asistían a la unidad de insuficiencia cardiaca y recibían atención multidisciplinaria, 33 de estos pacientes pertenecieron al grupo 1, 32 pacientes pertenecieron al grupo 2 y 32 pacientes pertenecieron al grupo 3 (tabla 1).

\section{Caracterización sociodemográfica}

En relación con la edad promedio de los participantes se encontró que fue de 62,46 años (desviación estándar 11,504, mínima 23, máxima 80 ), en cuanto al sexo predominó el masculino. En escolaridad se encontró que el nivel educativo que predominó fue el bachillerato y el universitario, seguido por la primaria y el técnico. El cuanto a la ocupación el mayor número fueron pensionados(as) y la mayor parte de los pacientes refirieron estar casados(as), este comportamiento fue similar en los tres grupos del estudio.

Se encontró como principal causa de la insuficiencia cardiaca la cardiopatía isquémica, seguida por la cardiopatía valvular y la categoría otras que hace referencia principalmente a la cardiopatía mixta (tabla 2).

\section{Resultados en la adherencia al tratamiento}

La evaluación inicial de la adherencia en los tres grupos, mostró que sus niveles medios son estadísticamente 
Tabla 1 Comportamiento de los pacientes en la muestra del estudio

\begin{tabular}{|c|c|c|c|}
\hline Muestra inicial & $\begin{array}{l}\text { Porcentaje } \\
\text { de pérdidas }\end{array}$ & Motivo & $\begin{array}{l}\text { Muestra } \\
\text { final }\end{array}$ \\
\hline Grupo 1 & $3,6 \%$ & Abandono programa $\mathrm{N}=1$ & Grupo 1 \\
\hline $\begin{array}{l}\text { Seguimiento telefónico más } \\
\text { mensajes texto } \\
N=37\end{array}$ & $N=4$ & $\begin{array}{l}\text { Alta programa } \mathrm{N}=1 \\
\text { No acepta la intervención } \\
\mathrm{N}=2\end{array}$ & $N=33$ \\
\hline Grupo 2 & $4,5 \%$ & Fallecimiento $\mathrm{N}=3$ & Grupo 2 \\
\hline $\begin{array}{l}\text { Seguimiento telefónico más } \\
\text { correos electrónicos } \\
\mathrm{N}=37\end{array}$ & $N=5$ & Alta programa $\mathrm{N}=2$ & $N=32$ \\
\hline Grupo 3 & $4,5 \%$ & ACV extenso $\mathrm{N}=1$ & Grupo 3 \\
\hline Control $\mathrm{N}=37$ & $N=5$ & $\begin{array}{l}\text { Deterioro cognitivo severo } \\
N=1 \\
\text { No responden pos test } N=2 \\
\text { Fallecimiento } N=1\end{array}$ & $N=32$ \\
\hline Total & $12,6 \%$ & & $N=97$ \\
\hline
\end{tabular}

Fuente. Base de datos de la investigación, 2015.

Tabla 2 Caracterización de la muestra

\begin{tabular}{|c|c|c|c|c|}
\hline & Total (n) & Grupo 1 (n) & Grupo 2 (n) & Grupo 3 (n) \\
\hline \multicolumn{5}{|l|}{ Sexo } \\
\hline Femenino & 42 & 13 & 16 & 13 \\
\hline Masculino & 55 & 20 & 16 & 19 \\
\hline \multicolumn{5}{|l|}{ Estado Civil } \\
\hline Soltero(a) & 11 & 4 & 3 & 4 \\
\hline Viudo(a) & 7 & 0 & 5 & 2 \\
\hline Divorciado(a) & 10 & 6 & 1 & 3 \\
\hline Casado(a) & 61 & 20 & 21 & 20 \\
\hline Unión libre & 8 & 3 & 2 & 3 \\
\hline \multicolumn{5}{|l|}{ Ocupación } \\
\hline Hogar & 21 & 8 & 6 & 7 \\
\hline Pensionado(a) & 34 & 9 & 16 & 9 \\
\hline Empleado(a) & 17 & 7 & 4 & 6 \\
\hline Independiente & 22 & 7 & 6 & 9 \\
\hline Desempleado(a) & 3 & 2 & 0 & 1 \\
\hline \multicolumn{5}{|l|}{ Escolaridad } \\
\hline Sabe leer y escribir & 7 & 1 & 4 & 2 \\
\hline Primaria & 18 & 5 & 6 & 7 \\
\hline Bachillerato & 25 & 10 & 6 & 9 \\
\hline Técnico & 14 & 4 & 5 & 5 \\
\hline Universitario & 24 & 9 & 7 & 8 \\
\hline Post grado & 9 & 4 & 4 & 1 \\
\hline \multicolumn{5}{|l|}{ Etiología } \\
\hline Isquémica & 41 & 16 & 12 & 13 \\
\hline Valvular & 21 & 7 & 5 & 9 \\
\hline Chagásica & 6 & 0 & 3 & 3 \\
\hline Idiopática & 6 & 3 & 2 & 1 \\
\hline Hipertensiva & 6 & 2 & 3 & 1 \\
\hline Otras & 17 & 5 & 7 & 5 \\
\hline
\end{tabular}

Fuente. Base de datos de la investigación, 2015. 
Tabla 3 Resultados de la prueba t pareada para la adherencia pre y pos intervención

\begin{tabular}{|c|c|c|c|c|c|c|c|c|}
\hline & \multicolumn{5}{|c|}{ Diferencias relacionadas } & \multirow[t]{3}{*}{$\mathrm{t}$} & \multirow[t]{3}{*}{$\mathrm{gl}$} & \multirow[t]{3}{*}{ Valor $p$} \\
\hline & \multirow[t]{2}{*}{ Media } & \multirow[t]{2}{*}{ Desviación } & \multirow[t]{2}{*}{ Error típico } & \multicolumn{2}{|c|}{ 95\% Intervalo de confianza para la diferencia } & & & \\
\hline & & & & Inferior & Superior & & & \\
\hline Grupo 1 & $-15,303$ & 8,248 & 1,436 & $-18,228$ & $-12,378$ & $-10,658$ & 32 & $<0,001$ \\
\hline Grupo 2 & $-14,125$ & 7,106 & 1,256 & $-16,687$ & $-11,563$ & $-11,244$ & 31 & $<0,001$ \\
\hline Grupo 3 & $-10,031$ & 8,582 & 1,517 & $-13,125$ & $-6,937$ & $-6,612$ & 31 & $<0,001$ \\
\hline
\end{tabular}

Fuente. Base de datos de la investigación, 2015.

iguales puesto que el análisis de varianza utilizado así lo sugirió $(p=0,224)$, de esta manera se aseguró que los tres grupos fueran similares en sus comportamientos de adherencia previos. El nivel de adherencia basal se ubicó en un rango de frecuentemente adherido para los tres grupos.

La evaluación de los cambios de la adherencia en cada uno de los grupos se llevó a cabo mediante la utilización de la prueba t para muestras independientes en donde los tres grupos definidos para el estudio cambiaron significativamente sus niveles medios de adherencia (tabla 3 ).

Los puntajes medios de los grupos de intervención alcanzaron el rango de totalmente adheridos, mientras que el grupo control a pesar de incrementar su puntaje medio se mantuvo en el rango de frecuentemente adherido.

Se demostró que los dos grupos de intervención generaron diferencias estadísticamente significativas con respecto a las mediciones obtenidas en el grupo control, lo cual se evidencia con una $p$ inferior a 0,0001. Al comparar los grupos de intervención se encontró que no existe diferencia estadísticamente significativa entre ellos en cuanto a la adherencia (tabla 4).

\section{Discusión}

La insuficiencia cardiaca es una enfermedad que afecta a la población mundial, como resultado final de diferentes enfermedades cardiovasculares, en Colombia es evidente esta problemática, encontrándose dentro de las primeras diez causas de la morbilidad y la mortalidad ${ }^{5}$, con una prevalencia del 2 al 3\% que la constituye como una causa frecuente de ingresos al servicio de urgencias ${ }^{14,15}$. En este sentido, la principal causa de insuficiencia cardiaca identificada en los pacientes del estudio correspondió a la cardiopatía isquémica con un $42,2 \%$, esto se explica por el comportamiento que en la actualidad tienen las enfermedades cardiovasculares lideradas por la enfermedad isquémica del corazón ${ }^{14}$.

Este contexto ha despertado un gran interés a nivel mundial y local que ha dado lugar a la implementación de diferentes estrategias, muchas de las cuales incluyen la creación de equipos multidisciplinarios conformados por diferentes profesionales como: enfermeros(as), cardiólogos(as), psicólogos(as), nutricionistas, entre otros, quienes complementan el manejo integral requerido y demuestran así tener un impacto positivo en la adherencia al tratamiento farmacológico y no farmacológico ${ }^{7,18,19-21}$. Por su parte Achury reportó un cambio significativo en la adherencia al tratamiento farmacológico y no farmacológico de los pacientes posterior a la realización de una intervención educativa obteniendo un rango de frecuentemente adheridos ${ }^{13}$, de igual manera Rojas confirma estos resultados en pacientes con falla cardiaca que asisten a una Clínica de Falla Cardiaca en Bucaramanga ${ }^{17}$.

Además, de los beneficios reportados tras la vinculación de los pacientes a los grupos multidisciplinarios previamente mencionados, las tecnologías de la información y la comunicación como estrategias innovadoras en el cuidado de la enfermería ${ }^{8,16,19}$, comprenden una amplia gama de servicios, tecnologías y aplicaciones que permiten generar, organizar, almacenar, manipular y transmitir información por medio de la utilización de redes de comunicación que incluyen los computadores, las aplicaciones informáticas, el internet, el correo electrónico y la telefonía, esto las hace herramientas novedosas que facilitan la implementación de nuevos modelos de atención, a través de los cuales se establecen canales de comunicación que promueven una relación enfermera-paciente individualizada basada en la comunicación empática y recíproca, por medio de la cual es posible brindar alternativas de manejo como parte del régimen terapéutico, vincular a la familia, reconocer las dificultades y plantear estrategias de solución ante las situaciones presentadas.

Esto resulta relevante dado que en la actualidad la mayor disponibilidad de celulares ${ }^{22}$, facilita la entrega y la recepción de mensajes de texto motivacionales y de seguimiento enfocados al reconocimiento de los síntomas de alarma, control de peso, importancia del tratamiento farmacológico, efectos secundarios de los medicamentos, realización de actividad física, vacunación, y manejo del estrés, como parte del tratamiento de la enfermedad, lo cual genera una ventaja importante sobre la revisión de los correos electrónicos, que requieren en la mayoría de los casos de la disponibilidad de otros elementos como: los computadores, la conexión al internet y en algunos casos el acompañamiento por parte de una red de apoyo primaria para tener acceso a esta herramienta tecnológica.

Lo anterior concuerda con lo reportado en las investigaciones realizadas al respecto en relación al uso de los mensajes de texto, en donde una de ellas a través de una revisión sistemática evaluó las intervenciones mHealth utilizadas para facilitar la adherencia al tratamiento de enfermedades crónicas, describiendo los mensajes de texto como la herramienta más utilizada, ya que facilita la adherencia al tratamiento, y encontrando que pueden ser utilizados por personas con poca experiencia tecnológica, son de bajo costo y en la actualidad se integran a los 
Tabla 4 Resultados de la prueba de comparaciones múltiples para la adherencia posintervención

\begin{tabular}{|c|c|c|c|c|c|}
\hline \multirow[t]{2}{*}{ Grupos } & \multirow{2}{*}{$\begin{array}{l}\text { Diferencia } \\
\text { de medias }\end{array}$} & \multirow{2}{*}{$\begin{array}{l}\text { Error } \\
\text { típico }\end{array}$} & \multirow{2}{*}{$\begin{array}{l}\text { Valor } \\
\mathrm{p}\end{array}$} & \multicolumn{2}{|c|}{ Intervalo de confianza al 95\% } \\
\hline & & & & Límite inferior & Límite superior \\
\hline \multicolumn{6}{|l|}{ Seguimiento telefónico y mensajes texto } \\
\hline Seguimiento telefónico y correos electrónicos & 1,871 & 1,631 & ,763 & $-2,11$ & 5,85 \\
\hline Control & $9,215^{*}$ & 1,631 &, 0001 & 5,24 & 13,19 \\
\hline \multicolumn{6}{|l|}{ Seguimiento telefónico y correos electrónicos } \\
\hline Seguimiento telefónico y mensajes texto & $-1,871$ & 1,631 & ,763 & $-5,85$ & 2,11 \\
\hline Control & $7,344^{*}$ & 1,644 & 0001 & 3,34 & 11,35 \\
\hline
\end{tabular}

Fuente. Base de datos de la investigación, 2015.

servicios de salud con facilidad; por otra parte se describen dificultades por posibles desigualdades en el acceso a la tecnología por parte de los pacientes ${ }^{9}$. De igual manera, en un estudio realizado en los pacientes que recibían tratamientos antirretrovirales indicaron que los envíos de recordatorios con mensajes de texto semanales aumentaron el porcentaje de participantes que alcanzaron el $90 \%$ de adherencia al tratamiento antirretroviral en aproximadamente un 13 a $16 \%$ en comparación con ningún recordatorio. Se comprobó que estos recordatorios semanales también fueron eficaces en la reducción de la frecuencia de las interrupciones del tratamiento ${ }^{10}$. Finalmente, en una revisión de la literatura realizada por Vervlolet, se reporta que los recordatorios electrónicos y especialmente los mensajes de texto parecen integrarse fácilmente en la vida del paciente por ser una intervención sencilla para el paciente y el profesional de la salud ${ }^{11}$.

Con respecto a los correos electrónicos las investigaciones que han empleado esta herramienta han descrito las ventajas de su uso. Este es el caso de una revisión sistemática de Cochrane, que describe dentro de las principales ventajas: su fácil uso, bajo costo, permitiendo la supervisión, el seguimiento y el apoyo continuo y cercano de los pacientes con enfermedades crónicas, así mismo, mejoramiento en la calidad de la atención y la calidad de vida. En cuanto a las desventajas reportan las asociadas a la pérdida de la comunicación verbal con el personal de salud, pérdida del contacto y falta de interés por revisar los correos, posibilidad de ampliar las desigualdades en la salud a través de la brecha digital y la presencia de problemas tecnológicos asociados a un buzón lleno, pérdida de la conexión al servidor y cuestiones médico-legales asociadas al manejo de la información ${ }^{12}$.

Las intervenciones mencionadas en la presente investigación se asociaron a seguimiento telefónico dado que si bien estas herramientas (mensajes de texto y correos electrónicos) son novedosas, de bajo costo y mejoran la adherencia al tratamiento, por sí solas se pone en riesgo la comunicación entre el paciente y el personal de la salud, dependen del acceso al internet por parte de quien las recibe y puede existir falta de interés por revisar los mensajes ${ }^{12}$, en este contexto cuando se utilizan asociadas a una estrategia como el seguimiento telefónico se fomenta una relación terapéutica entre el paciente y el profesional de enfermería que favorece la comunicación, el seguimiento y la retroalimentación de acuerdo a la necesidad de cada paciente.
La ventaja de implementar el seguimiento telefónico está descrita en la literatura; el estudio DIAL por ejemplo, reportó que con su uso fue posible reducir las hospitalizaciones totales y por causa cardiovascular, se mejoró la calidad de vida de los enfermos y se disminuyeron los costos de la atención médica, incluyendo a los pacientes ambulatorios con la insuficiencia cardiaca crónica estable. Todos fueron seguidos y tratados por médicos especialistas en cardiología que debían optimizar el tratamiento farmacológico previo al ingreso de acuerdo con las recomendaciones de las guías actuales $^{23}$, siendo la población con similares características a la del presente estudio. De igual manera, otros estudios confirman estos resultados, por ejemplo: el realizado por Cleland y colaboradores donde compararon tres grupos de pacientes, uno recibió telemonitorización con dispositivos de monitoreo de peso, presión arterial, ritmo cardiaco, otro recibió seguimiento telefónico por profesionales de enfermería y el tercer grupo recibió la atención habitual por médicos de atención primaria. Los resultados reportaron que los grupos de telemonitorización y seguimiento telefónico disminuyeron la mortalidad y los ingresos hospitalarios en comparación con la atención habitual; entre los grupos de telemonitoreo y seguimiento telefónico realizado por los profesionales de enfermería no existieron diferencias significativas ${ }^{24}$. Estos resultados son respaldados por una revisión de Cochrane en la cual se reportaron los beneficios de estas intervenciones, seguimiento telefónico estructurado y telemonitorización, en relación a la mortalidad, las hospitalizaciones, la duración de la estancia, la calidad de vida, la aceptabilidad, la capacidad funcional y los costos ${ }^{25}$.

Por su parte Riegel, comparó un grupo de pacientes que recibieron una intervención telefónica estandarizada durante 6 meses frente a los que recibían atención habitual, demostrando que la intervención telefónica utilizada lograba una reducción en los costos, recursos empleados en la atención y reducción en las hospitalizaciones ${ }^{26}$. El seguimiento telefónico sugiere otros beneficios como la identificación temprana de descompensaciones de los pacientes con insuficiencia cardiaca, así lo describe Romero, en un programa de seguimiento telefónico realizado por los profesionales de enfermería en una Unidad de Insuficiencia Cardiaca en Sevilla-España, donde concluye que es una herramienta eficaz y fácil para evaluar la estabilidad clínica del paciente, adicionalmente, permite el control e impacto del tratamiento farmacológico en los pacientes con insuficiencia cardiaca ${ }^{27}$. 
Todo lo anterior, respalda los resultados obtenidos en la adherencia al tratamiento farmacológico y no farmacológico de los pacientes que participaron de la presente investigación demostrando como el seguimiento telefónico asociado a las herramientas digitales como el mensaje de texto y los correos electrónicos tiene un impacto positivo en la adherencia al tratamiento, a través de la motivación y seguimiento de los pacientes, facilitando la comunicación no presencial de los pacientes con los profesionales de enfermería, que les permitió establecer una relación terapéutica efectiva y a través de la cual se hacía posible un mayor entendimiento de los contenidos educativos en relación al entendimiento de su enfermedad y su tratamiento farmacológico y no farmacológico.

\section{Conclusiones}

Conjugar los mensajes de texto y los correos electrónicos con estrategias como el seguimiento telefónico incrementan la adherencia al tratamiento, estableciendo canales de comunicación efectivos entre los pacientes y los profesionales de enfermería, lo que proporciona seguridad y motivación en los pacientes y facilita un mayor entendimiento y realización de las recomendaciones y comportamientos de autocuidado requeridos en respuesta a las alteraciones de su estado de salud.

Las mediciones basales ubicadas en un aceptable nivel de adherencia hacen visible el resultado del manejo multidisciplinario en unidades de insuficiencia cardiaca, en las cuales se intervienen algunas de las dimensiones que la determinan.

Una intervención de enfermería utilizando el seguimiento telefónico asociado a las tecnologías móviles permite obtener un nivel elevado de adherencia al tratamiento, facilitando el entendimiento de su enfermedad, su control, manejo de síntomas, facilita detección y descompensaciones, seguimiento y motiva a los pacientes a realizar sus comportamientos de autocuidado.

Para los pacientes con insuficiencia cardiaca, seguir el tratamiento de su enfermedad es complejo, por tanto, el profesional de enfermería debe acompañar al paciente a adquirir los conocimientos o habilidades relacionadas con su autocuidado, el uso de las tecnologías de la información y la comunicación en la práctica de enfermería se convierte en un sistema de apoyo educativo efectivo, en donde la enfermera(ro) como agente de cuidado terapéutico conduce al paciente con la insuficiencia cardiaca a adoptar una actitud responsable de su autocuidado, así se incrementa la adherencia.

Se sugiere la realización de nuevas investigaciones que permitan determinar el tiempo de seguimiento necesario para mantener el nivel de adherencia al tratamiento logrado posterior a la intervención.

Demostrar que estas herramientas tecnológicas se vislumbran como un modelo de atención de los pacientes con la insuficiencia cardiaca, que pueden ser generalizables a otras poblaciones a partir de los ensayos clínicos que demuestren su efectividad.

\section{Responsabilidades éticas}

Protección de personas y animales. Los autores declaran que para esta investigación no se han realizado experimentos en seres humanos ni en animales.

Confidencialidad de los datos. Los autores declaran que en este artículo no aparecen datos de pacientes.

Derecho a la privacidad y consentimiento informado. Los autores declaran que en este artículo no aparecen datos de pacientes.

\section{Conflicto de intereses}

Los autores manifiestan que no hay conflicto de intereses.

\section{Reconocimientos y agradecimientos}

A la Dirección de Investigación. Sede Bogotá, Programa Nacional de Apoyo a Estudiantes de Posgrado para el Fortalecimiento de la Investigación, código 201010021663.

A los participantes del estudio, al Equipo Multidisciplinario de la Unidad de Insuficiencia Cardiaca, al Cuerpo docente de la Facultad de Enfermería de la Universidad Nacional de Colombia y a la Fundación Universitaria Sanitas.

A la Unidad de Insuficiencia Cardiaca de la Clínica Colombia por su apoyo permanente para desarrollar este estudio. Al cuerpo docente de la Facultad de Enfermería de la Universidad Nacional de Colombia por su apoyo y acompañamiento en la realización de este estudio.

\section{Fuentes de apoyo}

Dirección de Investigación. Sede Bogotá Programa Nacional de Apoyo a Estudiantes de Posgrado para el Fortalecimiento de la Investigación, código 201010021663.

\section{Bibliografía}

1. Chaves WG, Diaztagle JJ, Sprockel JJ, Hernandez JI, Benavidez JM, Henao DC, et al. Factores asociados a mortalidad en pacientes con falla cardiaca descompensada. Acta Med Colomb. 2014;39(4):314-20.

2. Hernández-Leiva E. Epidemiología del síndrome coronario agudo y la insuficiencia cardiaca en Latinoamérica. Rev Española Cardiol. 2011;64 Supl 2:34-43.

3. McMurray JJ, Adamopoulos S, Anker SD, Auricchio A, Böhm M, Dickstein $\mathrm{K}$, et al. Guía de práctica clínica de la ESC sobre diagnóstico y tratamiento de la insuficiencia cardiaca aguda y crónica 2012. Rev Esp Cardiol. 2012;65:938.e1-59.

4. Conthe P, Tejerina F. Adhesión al tratamiento y calidad de vida en los pacientes con insuficiencia cardiaca. Rev Española Cardiol Supl. 2007;7:57F-66F.

5. Organización Panamericana de la Salud. Cardiovascular diseases mortality by major groups of causes. 2007. Disponible en: $w w w$. paho.org/hq/index.php?option=com_content\&view= article\&id $=6683 \&$ Itemid $=2391$ \&lang=es

6. Van Der Wal MHL, Jaarsma T. Adherence in heart failure in the elderly: Problem and possible solutions. Int J Cardiol. 2008; 125:203-8. 
7. McAlister FA, Stewart S, Ferrua SMJ. Multidisciplinary strategies for the management of heart failure patients at high risk for admission: A systematic review of randomized trials. Journal of the American College of Cardiology. 2004:810-9.

8. Miguel Morales J, Sarriá A. Modelos alternativos de atención para pacientes con insuficiencia cardiaca: revisión sistemática. Rev Calid Asist. 2006;21:51-60.

9. Hamine S, Gerth-Guyette E, Faulx D, Green B, Ginsburg A. Impact of mHealth chronic disease management on treatment adherence. J Med Internet Res. 2015;17:e52.

10. Pop-Eleches C, Thirumurthy H, Habyarimana JP, Zivin JG, Golds tein MP, de Walque D, et al. Mobile phone technologies improve adherence to antiretroviral treatment in a resource-limited setting: a randomized controlled trial of text mesage reminders. Aids. 2013;25:825-34.

11. Vervloet M, Linn a. J, van Weert JCM, de Bakker DH, Bouvy ML, van Dijk $L$. The effectiveness of interventions using electronic reminders to improve adherence to chronic medication: a systematic review of the literature. J Am Med Informatics Assoc. 2012;19:696-704.

12. Atherton H, Sawmynaden P, Sheikh A, Majeed ACJ. Email for clinical communication between patients/caregivers and healthcare professionals. Cochrane Database Syst Rev. 2012;14: CD007978, http://dx.doi.org/10.1002/14651858.CD007978. pub2

13. Achury DMS. Autocuidado y adherencia en pacientes con falla cardiaca. Aquichan. 2007;7:139-60.

14. Jaramillo C, Gómez E, Hernández E, Saldarriaga C, Flórez $\mathrm{N}$, Buitrago $\mathrm{R}$, et al. Consenso colombiano para el diagnostico y tratamiento de la Insuficiencia cardiaca crónica. Sociedad C. Bogotá; 2014, http://scc.org.co/blog/2015/01/ consenso-colombiano-parael-diagnostico-y-tratamiento-de-lainsuficiencia-cardiacaaguda-y-cronica/

15. Tamayo DC, Rodríguez VA, Rojas MX, Rincon M, Franco C, Ibarra MT, et al. Costos ambulatorios y hospitalarios de la falla cardiaca en dos hospitales de Bogotá. Acta Médica Colomb. 2013;38(4):208-12.

16. Cañon W, Orostegui A. Intervenciones educativas de enfermería en pacientes ambulatorios con falla cardiaca. Enferm Glob. 2013;12:52-67.

17. Rojas L, Echeverria L, Camargo F. Adherencia al tratamiento farmacológico y no farmacológico en pacientes con falla cardiaca Adherence to phararmacological and non-pharmacological treatment in patients with heart failure. Enferm glob. 2014;13:1-19.

18. Sabate E. Adherencia a los tratamientos a largo plazo. Pruebas para la acción. Organizacion Mundial de la Salud. 2004.

19. Güemes Careaga I. Telemonitorización en pacientes con insuficiencia cardiaca o enfermedad obstructiva crónica. Revisión sistemática de la literatura. Informe de Evaluación de Tecnologías Sanitarias. 2012. p. No EKU I12-01.

20. Holland R, Battersby J, Harvey I, Lenaghan E, Smith J, Hay L. Systematic review of multidisciplinary interventions in heart failure. Heart. 2005;91:899-906.

21. Lupón J, Parajón T, Urrutia A, González B, Herreros J, Altimir $\mathrm{S}$, et al. Reducción de los ingresos por insuficiencia cardiaca en el primer año de seguimiento en una unidad multidisciplinaria. Rev Española Cardiol. 2005;58:374-80.

22. Orozco M, Mazorra D, Foote J, Hopke J, Kim J, Tsang S, et al. Boletín de Prensa, Encuesta Comunicación Colombia 2012 Nuevas Tecnologías de Comunicación. Universidad Externado de Colombia Centro de Investigación en Comunicación Política. 2012:1-18.

23. Zambrano C, Badra R, Cerezo G, Ferrante D, Soifer S, Varini $S$, et al. Seguimiento al año luego de finalizada la intervención telefónica en pacientes con insuficiencia cardiaca crónica: estudio DIAL. Rev Argent Cardiol. 2005;73: 7-14.

24. Cleland J, Louis A, Rigby A, Janssens U, Balk A. Noninvasive home telemonitoring for patients with heart failure at high risk of recurrent admission and death: The TransEuropean Network-Home-Care Management System (TEN-HMS) study. JACC. 2005;45:1654-64.

25. Inglis S, Clark R, McAlister F, Ball J, Lewinter C, Cullington $D$, et al. Structured telephone support or telemonitoring programmes for patients with chronic heart failure. Cochrane Database Syst Rev. 2010:CD007228, http://dx.doi.org/10.1002/ 14651858.CD007228.pub2

26. Riegel B, Carlson B, Kopp Z, LePetri B, Glaser D, Unger A. Effect of a Standardized Nurse Case-Management Telephone Intervention on Resource Use in Patients With Chronic Heart Failure. Arch Intern Med. 2002;162:705-11.

27. Romero Rp, Ferraz C, Emma M, García G, Tomillero E. Diseño y características de un programa de seguimiento telefónico liderado por enfermería en una unidad de insuficiencia cardiaca. Rev Esp Cardiol. 2012;65:485. 